\title{
Deceptions in Quasicrystal Growth ${ }^{\star}$
}

\author{
Steven Dworkin, Jiunn-I Shieh \\ Department of Mathematics, The University of Texas, Austin, TX 78712-1082, USA \\ (e-mail: dworkin@math.utexas.edu jishieh@math.utexas.edu)
}

Received: 13 December 1993/in revised form: 6 June 1994

\begin{abstract}
We discuss a new general phenomenon pertaining to tiling models of quasicrystal growth. It is known that with Penrose tiles no (deterministic) local matching rules exist which guarantee defect-free tiling for regions of arbitrary large size. We prove that this property holds quite generally: namely, that the emergence of defects in quasicrystal growth is unavoidable for all aperiodic tiling models in the plane with local matching rules, and for many models in $\mathbf{R}^{\mathbf{3}}$ satisfying certain conditions.
\end{abstract}

\section{Introduction}

In 1984 Shechtman, Blech, Gratias, and Cahn [1] discovered quasicrystals, a new form of matter which exhibits an electron diffraction pattern with remarkable icosahedral symmetry. This symmetry is extraordinary because icosahedral symmetry is incompatible with atomic periodicity and therefore cannot exist for periodic crystals. To account for the unusual diffraction patterns of quasicrystals various models of their atomic structure have been proposed. Of these, the most extensively studied have been aperiodic tiling models (also known less precisely as deterministic quasicrystalline tilings) based on the two and three dimensional Penrose tiles and their variants (see for example Levine and Steinhardt [2]; Steinhardt and Ostlund [3]; Jarić [4]; Senechal [5]. Aperiodic tilings are defined in detail in Sect. 2.) In these tilings, points associated with each tile, perhaps the vertices, correspond to the centers of the atomic scatterers, and (deterministic) local matching rules designate how the tiles are to fit one next to the other.

To fix ideas consider the two dimensional Penrose model. In this model the two basic (proto) tiles are the kite and the dart (see Fig. 1). Along the four edges of each tile there are various "bumps" and "dents" which encode the local matching rules: two tiles match along a given edge provided they fit flush along that edge without gaps or overlap, much like the pieces of a jigsaw puzzle. A tiling of the plane (in

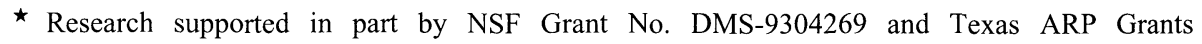
003658113 and 003658007 . 


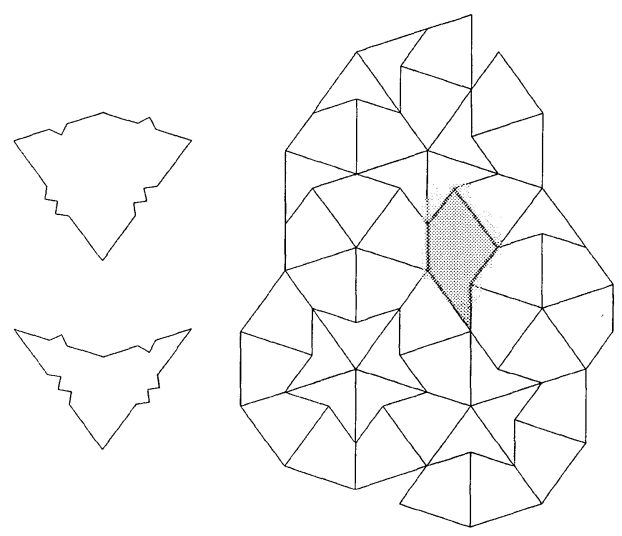

Fig. 1. Left: A Kite (above) and a dart (below). Right: A portion pf the plane tiled with kites and darts. The shaded region is a defect (hole) which can be filled by tiles satisfying the local matching rules

the case of the two dimensional Penrose model) consists then of a collection of tiles which covers the plane, wherein all pairs of tiles which touch must satisfy the local matching rules. It should be noted that given a tiling model the (deterministic) local matching rules can always be encoded as a series of bumps and dents along the edges of the prototiles (prototiles are the basic tile shapes of the tiling model, tiles are isometric copies of the prototiles; see Sect. 2 for further details).

Tiling models have, among other things, addressed two basic issues concerning quasicrystals: their structure and their growth.

As to the first, success has been achieved in reproducing the Bragg peaks in the diffraction patterns obtained by scattering of electrons, X-rays and neutrons $[2,6,7]$. This success has largely contributed to the popularity of these tilings as structural models for the positions of the atomic scatterers in quasicrystals, without addressing the important questions concerning the possible details of quasicrystal growth.

The second, more difficult question of quasicrystal growth has been more problematic. Typically, a tiling model with local matching rules models growth wherein tiles are added by accretion to an already existing patch of tiles provided they satisfy the local matching rules. (By a patch we mean a finite collection of tiles satisfying the local matching rules.) The problem has been one of trying to design such tiling models with local matching rules which can account for the long range order of quasicrystals. However it seems that with such aperiodic tiling models it is difficult to produce defect-free quasicrystals which cover the whole space. Without this ability one cannot guarantee the growth of perfect single grain quasicrystals of arbitrary large size. Simple placement of tiles one after the other (the correct matching rules being assumed) to a growing patch of tiles often leads to a quasicrystal which cannot be extended to a tiling of space.

For example, it has long been known that the two dimensional Penrose model could not guarantee the growth of perfect single grain quasicrystals of arbitrary large size (see for example Gardner [8]; Onoda, Steinhardt, DiVincenzo, and Socolar [9]; Jarić and Ronchetti [10]; Onoda et. al. [11]; Ingersent and Steinhardt 
[12]; Penrose [13]; Socolar [14]). If one naively builds up a patch by simply adding kites and darts one after the other, as a jigsaw puzzle might be assembled, a configuration is soon reached which cannot be extended to a tiling of the plane. There are two ways by which this may be manifest. The first, of less physical interest, is defects within the patch itself, consisting of "gaps" or "holes" which cannot be filled by kites or darts. The decapod seed cluster is one such defect in the Penrose model. It is important to note that local matching rules (in general) do allow the possibility of holes being filled whenever possible. For example, when adding kites and darts to a given patch we allow ourselves to lift the tiles off the table as it were - they do not have to push their way through other tiles on the table in order to gain access to the hole (see Fig. 1). Although not physically realistic, permitting such a possibility allows us to prove that the second kind of obstruction to a perfect quasicrystal tiling always exists under certain conditions.

This other kind of obstruction without defects we call a deception. A "deception of order $r "$ (defined more precisely in Sect. 2 below) is a patch without holes, not belonging to any tiling of the space, for which we cannot decide by local inspection (looking around within a distance $r$ ) whether the patch itself belongs to some tiling of the space. In other words a deception of order $r$ is a defect free patch which cannot be extended to a tiling of the space, but for which any subpatch of diameter less than $2 r$ belongs to some tiling of the space. We have then the following suggestive interpretation. Considering the local matching rules as nearest neighbour interactions between tiles, local inspection (within a distance $r$ ) is tantamount to a finite range interaction amongst tiles: a tile may be added to a patch provided every subpatch of diameter less than $2 r$ containing it belongs to some tiling of the space. Thus the existence of deceptions of order $r$ would imply that augmentation of the local matching rules to a finite range interaction of distance $r$ would be insufficient to guarantee the growth of defect-free patches of arbitrary large size. This phenomenon was known to be true for the two dimensional Penrose model [13].

In recognition of the problem of modelling defect-free quasicrystal growth, various modifications to the usual models have been suggested. For instance, Onoda, Steinhardt, Divincenzo, and Socolar $[9,14]$ added an additional probabilistic element to the usual local matching rules. In these models tiles are not added simply by accretion, but according to some preassigned probabilities, so as to ensure a high probability for the growth of perfect quasicrystals of limited grain size.

All the tiling models considered thus far have centered upon the Penrose tilings and its variants. Attempts to overcome the growth problem have lead to the introduction of various additional features to the basic aperiodic tiling models with local matching rules. A question which naturally arises is whether or not aperiodic tiling models with local matching rules (in the absence of any additional features such as probabilistic interactions), other than those based on the Penrose tiles and its variants, could perhaps eliminate the difficulties encountered with the growth problem. The answer is no; in this work we demonstrate that for all aperiodic tiling models of the plane, the emergence of defects in quasicrystal growth is unavoidable. Indeed, we prove that for all aperiodic tilings of the plane it is impossible, using only local matching rules, to guarantee the growth of perfect single grain quasicrystals of arbitrary large size. In addition, we prove that under certain conditions this is also true for aperiodic tilings of $\mathbf{R}^{\mathbf{3}}$. 


\section{Basic Definitions and Notation}

By a tiling space $V(S)$ of $\mathbf{R}^{\mathbf{m}}$ we mean a representation of $\mathbf{R}^{\mathbf{m}}$ as a union of "tiles" where:

a) there is a fixed finite set $S=\left\{p_{1}, p_{2}, \ldots, p_{\eta}\right\}$ of "prototiles," which are pairwise noncongruent homeomorphs of the closed $m$-ball;

b) each tile is an isometric copy of some prototile, that is, it is the image of a prototile by a symmetry operation (translation and/or rotation);

c) the interiors of the tiles do not overlap; and

d) given a tile, its boundary can be covered by other tiles, with no overlapping, in only a finite number of ways.

Loosely speaking, prototiles fit together much in the same way as jigsaw puzzle pieces. Again we emphasize that we do not restrict the placement of tiles to the exterior surface of a patch; holes may be filled if the local matching rules are not violated. A tiling is nonperiodic if it admits no translational symmetry other than the identity. Tiling models of the atomic structure of quasicrystals involve sets of prototiles which force nonperiodic tilings of the plane. We call $S$ an aperiodic protoset if each tiling in the (nonempty) set $V(S)$ is nonperiodic. As an abuse of terminology, $V(S)$ is sometimes referred to as an aperiodic tiling (space), or a quasicrystalline tiling. The most familiar example of an aperiodic protoset is the set of two Penrose (proto)tiles $[8,15]$.

By a patch we mean a finite set of nonoverlapping tiles. As mentioned above, with Penrose tiles it is impossible to decide by local inspection whether a patch belongs to some tiling of the plane [13]. This leads us quite naturally to embody this fact in the following notion of a "deception."

Let us first introduce some technical definitions. A patch will be regular if it is a homeomorph of the closed $m$-ball, and is of order $r$ if it covers some disc of radius $\tilde{r} \geqq \max \{r, 3 r \Delta\}$, where $\Delta=\sup _{i}\left\{\operatorname{diam}\left(p_{l}\right)\right\}$ (where the diameter of a subset $W \subseteq \mathbf{R}^{\mathbf{m}}$ is given by $\left.\operatorname{diam}(W)=\sup _{w_{1}, w_{2} \in W}\left\|w_{1}-w_{2}\right\|\right)$. Regular patches serve as models of perfect single grain quasicrystals. A regular patch $P$ of order $r$ will be a deception of order $r$ if:

a) every connected subpatch of $P$ of cardinality less than $r$ is a subset of some tiling of the plane, and

b) $P$ is not a subset of any tiling of the plane.

In other words, a deception of order $r$ is a regular patch of order $r$ for which local information (i.e. subpatches of tilings of the space) of "size" $\leqq r$ is insufficient to guarantee that the patch itself belongs to some tiling of the space.

In this paper we provide a criterion for aperiodic protosets of $\mathbf{R}^{\mathbf{3}}$ which guarantees the existence of deceptions of order $r$; further, we prove that each aperiodic protoset of the plane, $\mathbf{R}^{2}$ admits deceptions of all orders. This latter result demonstrates that, at least in the plane, no amount of local information is enough to guarantee that a given regular patch belongs to some tiling of the plane. In other words, no choice for local matching rules can guarantee perfect crystal growth in aperiodic tiling models.

For the proofs of the following theorems we will need to introduce the following definitions and notation.

Let $W$ be a subset of $\mathbf{R}^{\mathbf{m}}$. Denote the translation of $W$ by a vector $t \in \mathbf{R}^{\mathbf{m}}$ by $T^{t} W=\{t+w \mid w \in W\}$. For $W$ a subset of the plane, $\mathbf{R}^{2}$, denote the rotation of 
$W$ about the origin by an angle $\alpha \in \mathbf{R}$ by $R^{\alpha} W$. Given a patch $P$ which covers $W$, $P$ is a minimal patch covering $W$ if no subpatch of $P$ covers $W$. Given two regular patches $A$ and $B$, and a subset $W$ of $\mathbf{R}^{\mathbf{m}}$, let $C$ be a minimal simply connected patch covering $W$ such that $A \cup B \cup C$ is a regular patch. Then we call $C$ a matchmaker between $A$ and $B$ and covering $W$. A tiling $\Omega \in V(S)$ contains $s+1$ siblings of order $r$ if there exists a regular patch $A \subset \Omega$ or order $r$ such that:

a) there exist a set of vectors $\left\{t_{i} \mid t_{l} \in \mathbf{R}^{\mathbf{m}} ; i=0, \ldots, s\right\}$ such that $\left\{t_{i}-t_{0} \mid i=\right.$ $1, \ldots, s\}$ are $s$ linearly independent vectors in $\mathbf{R}^{\mathbf{m}}$

b) $\bigcup_{l=0}^{s} T^{t_{l}} A \subset \Omega$; and

c) $T^{t_{i}} A \cap T^{t_{l}} A=\emptyset$ for $0 \leqq i<j \leqq s$.

In particular we call a set of two siblings twins and a set of three siblings triplets. Given a patch $A$, we call the isometric patch $T^{t} R^{\beta} A$ for $t \in \mathbf{R}^{\mathbf{2}}, \beta \in(0,2 \pi)$ a cousin of $A$. For $\varepsilon>0$, siblings are $\varepsilon$-nice if $\frac{\left|\left\langle\left(t_{j}-t_{0}\right),\left(t_{t}-t_{0}\right)\right\rangle\right|}{\left\|t_{j}-t_{0}\right\|\left\|t_{t}-t_{0}\right\|}<\varepsilon$ for all $i \neq j$. Intuitively, siblings are $\varepsilon$-nice if pairs of vectors $\left\{\left(t_{i}-t_{0}\right),\left(t_{j}-t_{0}\right)\right\}$ are almost orthogonal for $i \neq j$.

We say that $V(S)$ admits a patch $W$ if there exists some $\Omega \in V(S)$ such that $W \subset \Omega$.

Let $B_{p}(r)$ denote the open ball of radius $\mathrm{r}$ centered at $p$, and $L_{a, b}$ denote the line segment from point $a \in A$ to point $b \in B$. Denote the right circular cylinder of radius $r$ and with axis $L_{a, b}$ by $\mathscr{C}\left(r, L_{a, b}\right)$.

We say a tiling enjoys the local isomorphism property if for every patch $P$ in the tiling there is some distance $r(P)$ such that for any point $p$ the ball $B_{p}(r(P))$ contains an isometric copy of the patch. Radin and Wolff [16] proved that if a set of prototiles admits a tiling of the space, it must admit a tiling satisfying the local isomorphism property (we use this fact in the proof of Theorem 2 below).

We say a nonempty closed translation invariant subset $X$ of $V(S)$ is minimal if there are no proper closed subset of $X$ which is invariant under translations.

\section{Results}

Lemma 1. Let $S=\left\{p_{i} \mid i=1, \ldots, \eta\right\}$ be a finite set of prototiles of $\mathbf{R}^{\mathbf{m}}$. Given a (finite) patch $P \subseteq B_{0}\left(r_{1}\right)$ and $r_{2} \geqq r_{1}$ there exists at most finitely many patches $\tilde{P}$ such that $B_{0}\left(r_{1}\right) \subseteq \tilde{P} \subseteq B_{0}\left(r_{2}\right)$ and $P \subseteq \tilde{P}$.

The proof is elementary.

Nice Lemma. Let $V(S)$ be a space of tilings of $\mathbf{R}^{\mathbf{m}}$ which admits $s+1$ siblings of order $r$. Then either $V(S)$ admits a deception of order $r$ or admits $s+1 \varepsilon$-nice siblings of order $r$, for any $\varepsilon>0$.

Proof. The proof will be by induction. Assume $\Omega \in V(S)$ contains $s+1$ siblings of order $r$, namely: $T^{t_{i}} A, i=0, \ldots, s$. Consider the sequence of regular patches of order $r$ given by:

$$
P_{n}=\bigcup_{J=0}^{s} \bigcup_{i_{j}=-n}^{n} T^{l^{\prime}\left(t_{j}-t_{0}\right)}\left[B_{j} \cup T^{t_{j}} A\right],
$$

where $B_{0}=\emptyset$ and $B_{i}$ is a matchmaker between $T^{t_{0}} A$ and $T^{t_{i}} A$ and covering the line segment $L_{t_{0}, t_{i}, i}=1, \ldots, s$. If for some $N \in \mathbf{N} P_{N} \not \subset \Omega^{\prime}$ for any $\Omega^{\prime} \in V(S)$, then $P_{N}$ 
is a deception of order $r$; else suppose $\left\{T^{t_{0}} A, \ldots, T^{t_{k-1}} A\right\}$ are $k \varepsilon$-nice siblings of order $r, k \geqq 1$. We will show it extends to $k+1 \varepsilon$-nice siblings of order $r$.

Consider the continuous function $f^{k}: \mathbf{R}^{\mathbf{k}} \backslash\{0\} \rightarrow \mathbf{R}$ given by

$$
f^{k}\left(x_{1}, \ldots, x_{k}\right)=\sum_{i=1}^{k-1} \frac{\left|\left\langle\left[x_{k}\left(t_{k}-t_{0}\right)-\sum_{j=1}^{k-1} x_{j}\left(t_{j}-t_{0}\right)\right],\left(t_{i}-t_{0}\right)\right\rangle\right|}{\left\|\left[x_{k}\left(t_{k}-t_{0}\right)-\sum_{j=1}^{k-1} x_{j}\left(t_{j}-t_{0}\right)\right]\right\|\left\|t_{i}-t_{0}\right\|}
$$

By the Gram-Schmidt orthogonalization process there exists $c_{i} \in \mathbf{R}, i=1, \ldots, k$ such that $f^{k}\left(c_{1}, \ldots, c_{k}\right)=0$, for $c_{i} \neq 0$ for some $i$. By continuity of $f^{k}$, given $\varepsilon>0$ there exists $\left\{a_{i}=p_{l} / q_{i} \mid p_{i} \in \mathbf{Z} \backslash\{0\}, q_{i} \in \mathbf{N}, i=1, \ldots k\right\}$ such that $\left|f^{k}\left(a_{1}, \ldots, a_{k}\right)\right|$ $<\varepsilon$. Take $m_{l}=a_{i} \prod_{j=1}^{k} q_{j} \in \mathbf{Z}$ for $i=1, \ldots, k, m_{0}=0$. Then $\left\{T^{\left(t_{0}+m_{i}\left(t_{i}-t_{0}\right)\right)} A \mid i\right.$ $=0, \ldots, k\}$ are $k+1 \varepsilon$-nice siblings of order $r$.

Q.E.D.

Theorem 1. Let $V(S)$ be a space of tilings of $\mathbf{R}^{3}$ with aperiodic prototile set $S$. If $V(S)$ admits 4 siblings of order $r$, then $V(S)$ admits a deception of order $r$.

Proof. The proof will be by contradiction.

Assume $V(S)$ does not admit deceptions of order $r$;

in particular every regular patch of order $r$ is a subset of some tiling in $V(S)$. By the Nice Lemma we may assume $\Omega \in V(S)$ contains $4 \varepsilon$-nice siblings of order $r$, namely: $A, T^{t_{i}} A, i=1,2,3$ and $A$ a regular patch of order $r$ containing $B_{0}(\tilde{r})$. Let $\Gamma_{0}=A \bigcup_{i=1}^{3}\left[T^{t_{i}} A \cup B_{i}\right]$ (see Fig. 4), where $B_{l}$ is a matchmaker between $A$ and $T^{t_{l}} A$ and covering the right circular cylinder of radius $r \Delta$ with axis $L_{0, t_{i}}, i=1,2,3$ (see Fig. 2). (This latter condition is necessary to ensure that when we join two "faces" at near right angles (as we do below), given a subpatch $P$ of cardinality $r$ bridging two faces, $P \subset \Omega^{\prime \prime}$ for some $\Omega^{\prime \prime} \in V(S)$ (see Fig. 3). The Nice Lemma is necessary to ensure the near perpendicularity of the "faces," and consequently simplifies certain calculations below.)

Step 1. We will prove that $V(S)$ admits a patch $F^{1}$ which can be extended periodically to cover a plane $\mathscr{Y}_{1,2}$, spanned by $\left\{t_{1}, t_{2}\right\}$. Intuitively, $F^{1}$ will serve as a temporary bottom to a certain "cube" we wish to construct.

Let $E^{1}=A \cup B_{1} \cup T^{t_{1}} A$ and $Z^{1}=E^{1} \cup T^{t_{2}} E^{1}$ (see Fig. 2 and Fig. 5). Consider the sequence of regular patches given by $\Gamma_{n}^{1}=\Gamma_{0} \bigcup_{i_{1}=0}^{n} T^{i_{1} t_{1}} Z^{1}$ (see Fig. 6).

(Although not used in the proof per se, these patches - and the $\Gamma_{n}^{j}$ to follow are given to assist the reader in following the constructions of the proof.) Let $H_{1,2}$ be the union of the convex hull of $\left\{0, t_{1}, t_{2}, t_{1}+t_{2}\right\}$ with the two cylinders $\mathscr{C}\left(r \Delta, L_{0, t_{2}}\right)$ and $T^{t_{1}}\left[\mathscr{C}\left(r \Delta, L_{0, t_{2}}\right)\right]$. Let $\mathscr{F}^{1}=\left\{F_{u}^{1} \mid u=1, \ldots, q_{1}\right\}$ denote the finite set of all minimal patches, $F_{u}^{1}$, covering $H_{1,2}$ such that $F_{u}^{1} \cup Z^{1}$ is a regular patch of order $r$ for all $u$. (Intuitively, we construct patches $F_{u}^{1}$ which roughly fill the space between the bounding sides of $Z^{1}$, and having "fat edges" covering right circular cylinders of radius $r \Delta$ (see Fig. 7.) That $\mathscr{F}^{1}$ is finite follows by Lemma 1. It follows from supposition (1) that there exists some map $u: \mathbf{N} \rightarrow\left\{1, \ldots, q_{1}\right\}$ such that $\bigcup_{i_{1} \in \mathbf{N}} T^{i_{1} t_{1}}\left[F_{u\left(l_{1}\right)}^{1} \cup Z^{1}\right] \subset \tilde{\Omega}$ for some $\tilde{\Omega} \in V(S)$ (see Fig. 8). Thus there exists $\lambda_{1}<\lambda_{1}^{\prime} \in \mathbf{N}$ such that $u\left(\lambda_{1}\right)=u\left(\lambda_{1}^{\prime}\right)$ (for given a sequence of $n$ symbols of length $m, m>n$, then at least two symbols must repeat). Let $F^{1}=\bigcup_{i_{1}=\lambda_{1}}^{\lambda_{1}^{\prime}-1} T^{\left(i_{1}-\lambda_{1}\right) t_{1}}\left[F_{u\left(i_{1}\right)}^{1} \cup Z^{1}\right]$. Then $F^{1}$ is a regular patch of order 


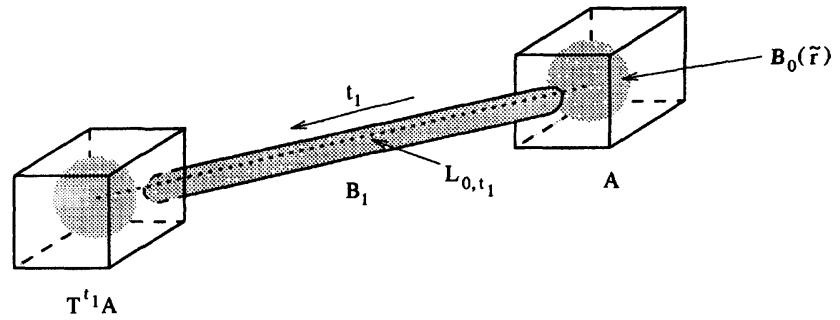

Fig. 2. $E^{1}$

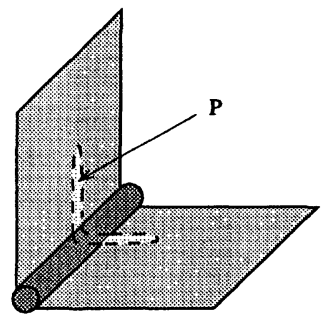

Fig. 3.

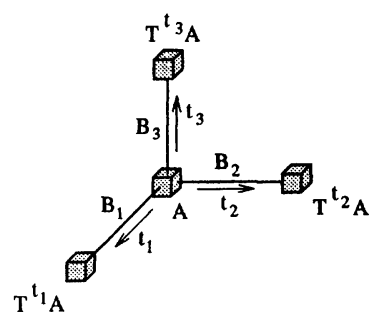

Fig. 4. $\Gamma_{0}$

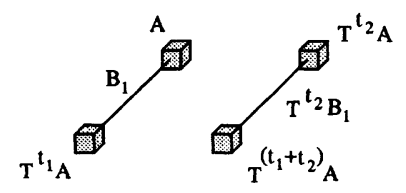

Fig. 5. $Z^{1}$

$r$ which, because opposite edges are identical, can be extended periodically to cover a plane $\mathscr{Y}_{1,2}$ spanned by $\left\{t_{1}, t_{2}\right\}$.

Step 2. We will prove that $V(S)$ admits a patch $F^{2}$, related in a certain way to $F^{1}$, which can be extended periodically to cover a plane $\mathscr{Y}_{2,3}$, spanned by $\left\{t_{2}, t_{3}\right\}$. 


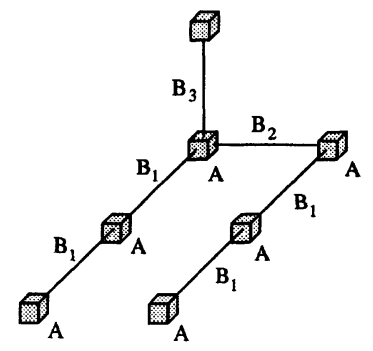

Fig. 6. $\Gamma_{2}^{1}$

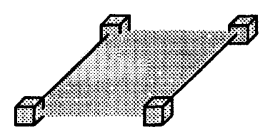

Fig. 7. $F_{u}^{1} \cup Z^{1}$

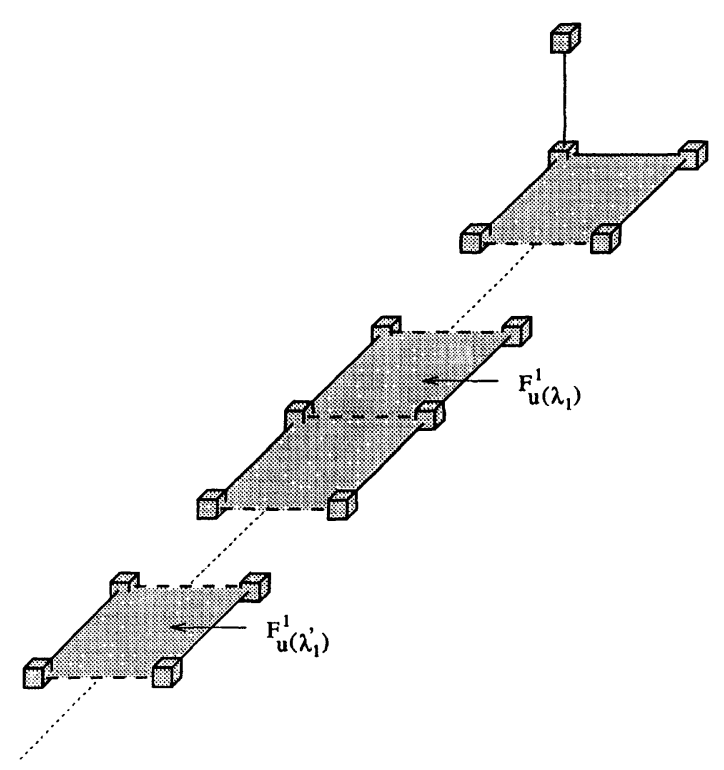

Fig. 8 .

Intuitively, $F^{2}$ will serve in the construction of the front and back faces to a certain "cube" we wish to construct.

Let $\tilde{B}_{2}$ be the matchmaker covering $\mathscr{C}\left(r \Delta, L_{0, t_{2}}\right)$ and contained in $F^{1}$. ( $\tilde{B}_{2}$ is a "fat edge" of $F^{1}$ parallel to $t_{2}$.) Let $E^{2}=A \cup \tilde{B}_{2} \cup T^{t_{2}} A$ and $Z^{2}=E^{2} \cup T^{t_{3}} E^{2}$. Consider the sequence of regular patches given by $\Gamma_{n}^{2}=B_{3} \bigcup_{l_{2}=0}^{n} T^{l_{2} t_{2}}\left[F^{1} \cup Z^{2}\right]$ (see Fig. 9). Let $H_{2,3}$ be the union of the convex hull of $\left\{0, t_{2}, t_{3}, t_{2}+t_{3}\right\}$ with the two cylinders $\mathscr{C}\left(r \Delta, L_{0, t_{3}}\right)$ and $T^{t_{2}}\left[\mathscr{C}\left(r \Delta, L_{0, t_{3}}\right)\right]$. Let $\mathscr{F}^{2}=\left\{F_{u}^{2} \mid u=1, \ldots, q_{2}\right\}$ denote 


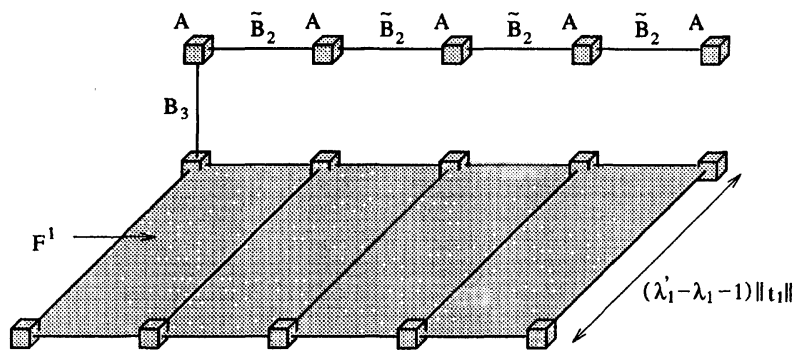

Fig. 9. $\Gamma_{4}^{2}$

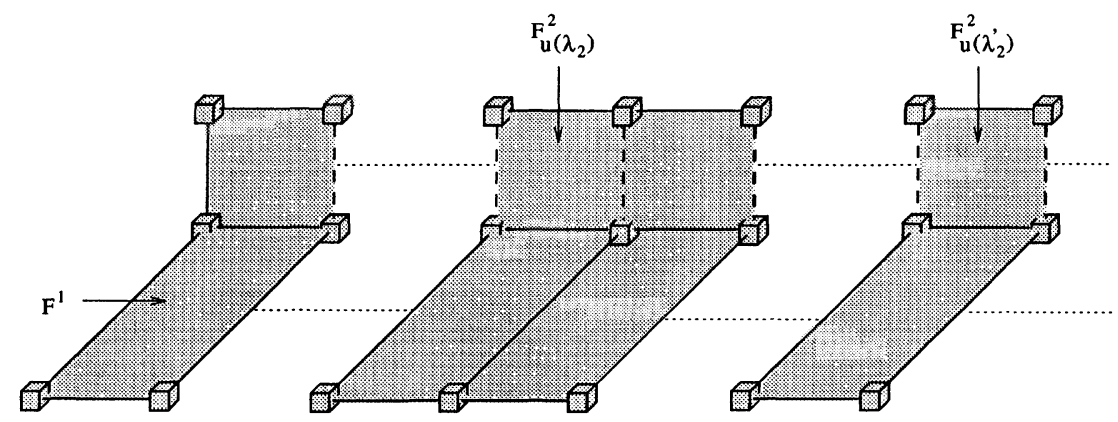

Fig. 10.

the finite set of all minimal patches, $F_{u}^{2}$, covering $H_{2,3}$ such that $F_{u}^{2} \cup Z^{2}$ is a regular patch of order $r$ for all $u$. (Intuitively, we construct patches $F_{u}^{2}$ which roughly fill the space between the bounding sides of $Z^{2}$, and having all four "fat edges" covering right circular cylinders of radius $r \Delta$.) It follows from (1) that there exists some map $u: \mathbf{N} \rightarrow\left\{1, \ldots, q_{2}\right\}$ such that $\bigcup_{i_{2} \in \mathbf{N}} T^{i_{2} t_{2}}\left[F^{1} \cup F_{u\left(l_{2}\right)}^{2} \cup Z^{2}\right] \subset \tilde{\Omega}$ for some $\tilde{\Omega} \in V(S)$ (see Fig. 10). Thus there exists $\lambda_{2}<\lambda_{2}^{\prime} \in \mathbf{N}$ such that $u\left(\lambda_{2}\right)=u\left(\lambda_{2}^{\prime}\right)$. Let $F^{2}=\bigcup_{l_{2}=\lambda_{2}}^{\lambda_{2}^{\prime}-1} T^{\left(i_{2}-\lambda_{2}\right) t_{2}}\left[F_{u\left(l_{2}\right)}^{2} \cup Z^{2}\right]$. Then $F^{2}$ is a regular patch of order $r$ which, because opposite edges are identical, can be extended periodically to cover a plane $\mathscr{Y}_{2,3}$, spanned by $\left\{t_{2}, t_{3}\right\}$.

Step 3. We will prove that $V(S)$ admits a patch $C$ which can be stacked periodically in the $t_{3}$ direction. Intuitively, $C$ will serve in the construction of the four side faces (front and back, and left and right) to a certain "cube" we wish to construct. $C$ will have the property that front and back faces will be identical, as will left and right faces.

Let $Z^{3}=F^{2} \cup T^{\left(\lambda_{1}^{\prime}-\lambda_{1}-1\right) t_{1}} F^{2}$ (front and back faces) and $\tilde{F}^{1}=\bigcup_{l_{2}=0}^{\left(\hat{\lambda}_{2}^{\prime}-\hat{\lambda}_{2}-1\right)} T^{i_{2} t_{2}} F^{1}$ (bottom face). Consider the sequence of regular patches given by $\Gamma_{n}^{3}=\tilde{F}^{1} \bigcup_{i_{3}=0}^{n}$ $T^{l_{3} t_{3}} Z^{3}$ (see Fig. 11). (It is necessary to include $\lambda_{2}^{\prime}-\lambda_{2}-1$ translates of $F^{1}, \tilde{F}^{1}$, so that the bottom face is of the same length in the $t_{2}$ direction as that of the front and back faces $Z^{3}$.) Let $H_{1,3}$ be the convex hull of $\left\{0,\left(\lambda_{1}^{\prime}-\lambda_{1}-1\right) t_{1}, t_{3}\right.$, $\left.\left(\lambda_{1}^{\prime}-\lambda_{1}-1\right) t_{1}+t_{3}\right\}$, and $\mathscr{F}^{3}=\left\{F_{u}^{3} \mid u=1, \ldots, q_{3}\right\}$ denote the finite set of all minimal patches, $F_{u}^{3}$, covering $H_{1,3}$ such that $F_{u}^{3} \cup Z^{3}$ is a regular patch of order $r$ for 


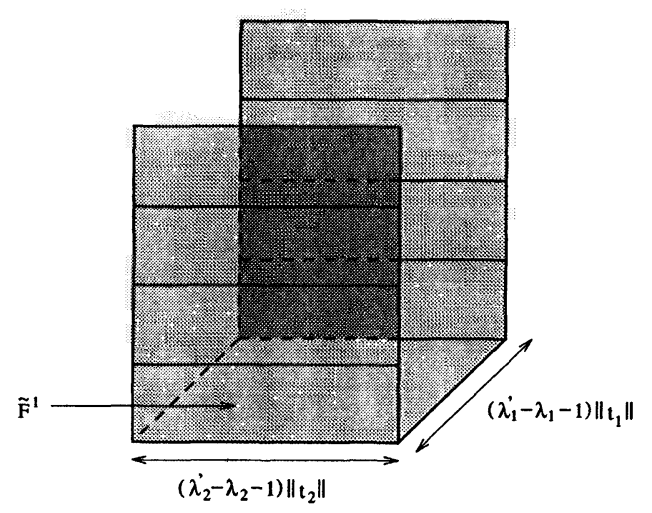

Fig. 11. $\Gamma_{4}^{3}$

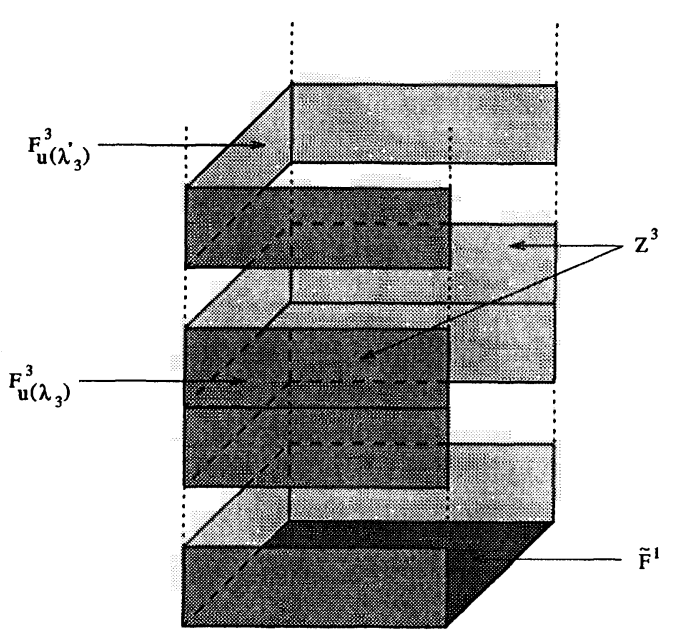

Fig. 12.

all $u$. (Intuitively, we construct side faces $F_{u}^{3}$ which meet the "edges" of $Z^{3}$ nicely.) It follows from (1) that there exists some map $u: \mathbf{N} \cup\{0\} \rightarrow\left\{1, \ldots, q_{3}\right\}$ such that $\tilde{F}^{1} \bigcup_{i_{3} \in \mathbf{N} \cup\{0\}} T^{i_{3} t_{3}}\left[F_{u\left(i_{3}\right)}^{3} \cup Z^{3}\right] \subset \tilde{\Omega}$ for some $\tilde{\Omega} \in V(S)$ (see Fig. 12). Thus there exists $\lambda_{3}<\lambda_{3}^{\prime} \in \mathbf{N}$ such that $u\left(\lambda_{3}\right)=u\left(\lambda_{3}^{\prime}\right)$. Let $Z_{u}^{4}=F_{u}^{3} \cup T^{\left(\lambda_{2}^{\prime}-\lambda_{2}-1\right) t_{2}} F_{u}^{3}$ (left and right faces) and let $C$ be the "collar" given by $C=\bigcup_{i_{3}=\lambda_{3}}^{\lambda_{3}^{\prime}-1} T^{i_{3} t_{3}}\left[Z^{3} \cup Z_{u\left(i_{3}\right)}^{4}\right]$ (see Fig. 13). $C$ permits a periodic stacking of itself in the $t_{3}$ direction; and right and left faces are identical, as are front and back faces.

Step 4. We will prove that $V(S)$ contains a "cube" $\Lambda$ which can be extended periodically to cover all of $\mathbf{R}^{3}$ 


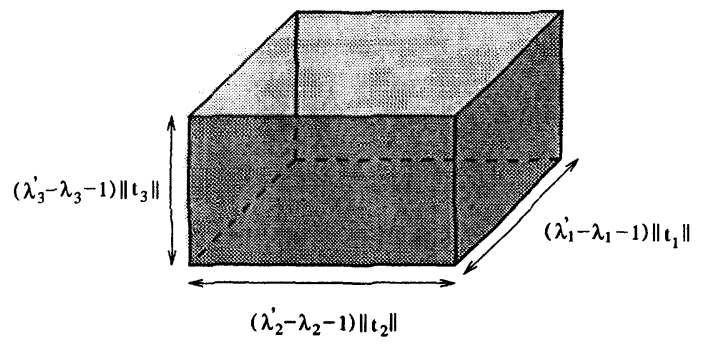

Fig. 13. $C$

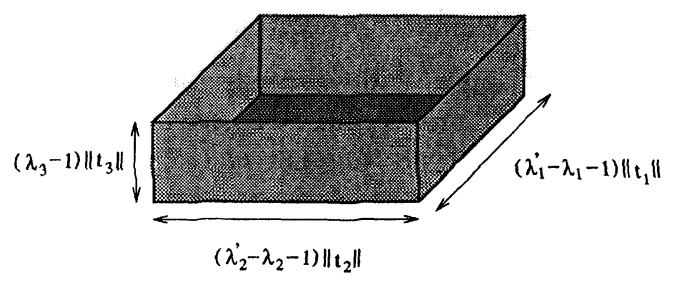

Fig. 14. $J$

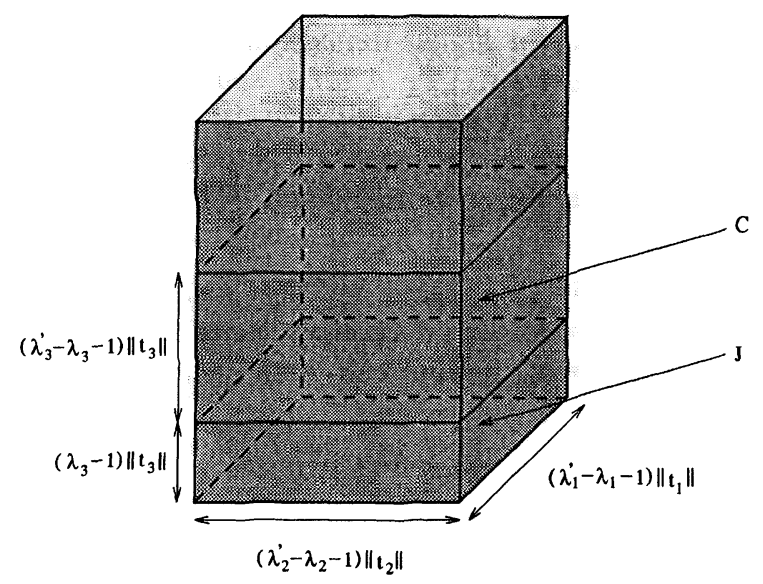

Fig. 15. $\Gamma_{2}^{4}$

Let $J=\tilde{F}^{1} \bigcup_{l_{3}=0}^{\left(\lambda_{3}-1\right)} T^{i_{3} t_{3}}\left[Z^{3} \cup Z_{u\left(i_{3}\right)}^{4}\right]$. ( $J$ is a "box without a top" (see Fig. 14).) Consider the sequence of regular patches given by $\Gamma_{n}^{4}=J \bigcup_{l_{3}=0}^{n} T^{i_{3}\left(\lambda_{3}^{\prime}-\lambda_{3}-1\right) t_{3}} C$ (see Fig. 15). Let $H_{1,2,3}$ be the convex hull of

$$
\begin{aligned}
& \left\{0,\left(\lambda_{1}^{\prime}-\lambda_{1}-1\right) t_{1},\left(\lambda_{2}^{\prime}-\lambda_{2}-1\right) t_{2},\left(\lambda_{3}^{\prime}-\lambda_{3}-1\right) t_{3},\right. \\
& \left.\quad \ldots,\left(\lambda_{1}^{\prime}-\lambda_{1}-1\right) t_{1}+\left(\lambda_{2}^{\prime}-\lambda_{2}-1\right) t_{2}+\left(\lambda_{3}^{\prime}-\lambda_{3}-1\right) t_{3}\right\}
\end{aligned}
$$


and $\mathscr{F}^{4}=\left\{F_{u}^{4} \mid u=1, \ldots, q_{4}\right\}$ denote the finite set of all minimal patches, $F_{u}^{4}$, covering $H_{1,2,3}$ such that $F_{u}^{4} \cup C$ is a regular patch of order $r$ for all $u$. (Intuitively, the patches $F_{u}^{4}$ are "solid blocks" which, roughly, fill the interior of collar C.) It follows from (1) that there exists some map $u: \mathbf{N} \rightarrow\left\{1, \ldots, q_{4}\right\}$ such that $\bigcup_{i_{3} \in \mathbf{N}} T^{l_{3}\left(\lambda_{3}^{\prime}-\lambda_{3}-1\right) t_{3}}\left[F_{u\left(i_{3}\right)}^{4} \cup C\right] \subset \tilde{\Omega}$ for some $\tilde{\Omega} \in V(S)$. Thus there exists $\lambda_{4}<\lambda_{4}^{\prime} \in \mathbf{N}$ such that $u\left(\lambda_{4}\right)=u\left(\lambda_{4}^{\prime}\right)$. Let $\Lambda=\bigcup_{l_{3}=\lambda_{4}}^{\lambda_{4}^{\prime}-1} T^{\iota_{3}\left(\lambda_{3}^{\prime}-\lambda_{3}-1\right) t_{3}}\left[F_{u\left(i_{3}\right)}^{4} \cup C\right]$. Then $\Lambda$ is a regular patch which, because all opposite faces are identical, can be extended periodically to cover the entire space $\mathbf{R}^{\mathbf{3}}$. This contradicts the fact that $S$ is an aperiodic protoset, and therefore $V(S)$ must admit a deception of order $r$.

Q.E.D.

Corollary. Let $V(S)$ be a tiling space of $\mathbf{R}^{\mathbf{3}}$ with aperiodic prototile set $S$. If there exists $\Omega \in V(S)$ such that $\Omega$ admits only finitely many orientations of the prototiles of $S$, then $V(S)$ admits deceptions of all orders.

Proof. Since $\Omega$ admits only finitely many orientations of the prototiles of $S$, each patch contained in $\Omega$ may occur with only finitely many orientations. Given $r>0$ let $\mathscr{P}$ be the set of all regular patches of order $r$ contained in some ball of fixed radius $R(R>\max \{2 r, 6 r \Delta\}$; see definition of regular patches of order $r$ ). It follows immediately that $\mathscr{P}$ has finite cardinality. Since there exist infinitely many mutually disjoint balls of radius $R$ in $\mathbf{R}^{\mathbf{3}}$, one of the patches belonging to $\mathscr{P}$ must occur infinitely often, not all the patches corresponding to this choice intersecting a fixed plane. Thus $\Omega$ admits 4 siblings of order $r$.

Q.E.D.

Remark 1. We believe the proof of Theorem 1 can be generalized quite naturally to prove a similar result for higher dimensional tiling spaces. Namely, we believe the following is true: Let $V(S)$ be a tiling space of $\mathbf{R}^{\mathbf{m}}$ with aperiodic prototile set $S$. If $V(S)$ admits $m+1$ siblings of order $r$, then $V(S)$ admits a deception of order $r$. The authors have not pursued a rigorous proof of this proposition as we believe the details would prove quite tedious. The corollary above would then also generalize accordingly.

Remark 2. Although it is possible for deceptions to exist in a periodic tiling space $V(S)$ of $\mathbf{R}^{\mathbf{m}}, m \geqq 2$, such deceptions cannot be of all orders. If $r$ is chosen larger than the diameter of the unit cell, then clearly deceptions of order $r$ do not exist.

Theorem 2. Any space of tilings $V(S)$ of the plane $\mathbf{R}^{\mathbf{2}}$ with aperiodic protoset $S$ admits deceptions of all orders.

Proof. The proof will be by contradiction.

Assume $V(S)$ does not admit deceptions of some order $r$;

in particular, every regular patch of order $r$ will belong to some tiling of the plane. Choose $\Omega \in V(S)$ belonging to some minimal subset $X \subseteq V(S)$, and let $A \subset \Omega$ be any regular patch of order $r$ containing $B_{0}(\tilde{r})$.

Step 1. We will prove that $V(S)$ admits twins and a cousin of order $r$. By the local isomorphism property (1.i.p.) of $X$ [16] there exists $A \cup T^{t_{1}} R^{\alpha} A \subset \Omega$ for $t_{1} \in \mathbf{R}^{2},\left\|t_{1}\right\|>[2 \operatorname{diam}(A)+1]$ (this condition guarantees that $A$ and $T^{t_{1}} A$ are disjoint), $\alpha \in\left(-\frac{\pi}{100}, \frac{\pi}{100}\right)$ (see Fig. 16). 


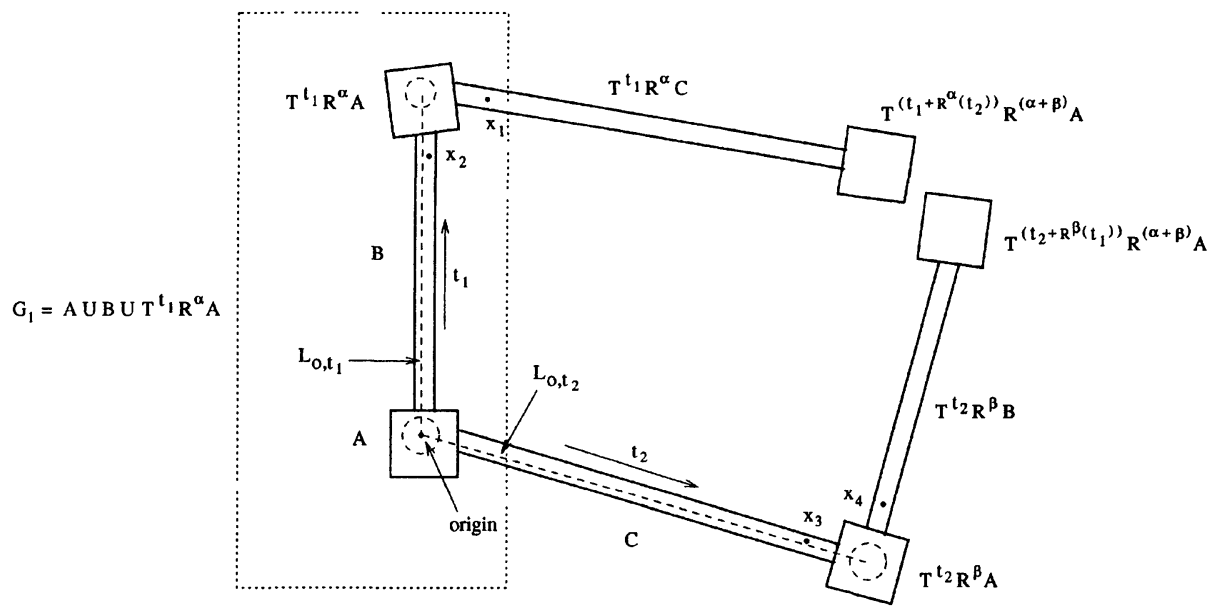

Fig. 16. $G_{2}$

If $\alpha=0$ then $\Omega$ has twins, and by another application of the 1.i.p. we may assume there exists a cousin $T^{t_{2}} R^{\beta} A \subset \Omega$ as well; we then proceed to Step 2 below.

If $\alpha \neq 0$ we may assume without loss of generality that $\alpha>0$ by interchanging the roles of $A$ with $T^{t_{1}} R^{\alpha} A, t_{1}$ with $-t_{1}$, and the origin with $t_{1}$. Let $B$ be a matchmaker between $A$ and $T^{t_{1}} R^{\alpha} A$ and covering the line segment $L_{0, t_{1}}$. Let $G_{1}=A \cup B \cup T^{t_{1}} R^{\alpha} A$ be the resultant regular patch in $\Omega$ (see Fig. 16).

Again by the 1.i.p. of $X$ there exists $T^{t_{2}} R^{\beta} G_{1} \subset \Omega$ such that:

a) $\beta \in\left(-\frac{\pi}{100}, \frac{\pi}{100}\right)$;

b) $T^{t_{2}} R^{\beta} G_{1} \cap G_{1}=\emptyset$;

c) $\left\|t_{2} \sin (\alpha)\right\|>3 \operatorname{diam}(A)$

d) $\cos ^{-1}\left(t_{1} \cdot t_{2} /\left\|t_{1}\right\|\left\|t_{2}\right\|\right) \in\left(\frac{\pi}{2}, \frac{3 \pi}{4}\right)$.

Let $C$ be a matchmaker between $A$ and $T^{t_{2}} R^{\beta} A$ and covering the line segment $L_{0, t_{2}}$. (Then $d\left(C, T^{-t_{1}} R^{-\alpha} B\right)>\tilde{r}$ and $d\left(B, T^{-t_{2}} R^{-\beta} C\right)>\tilde{r}$ by $(\mathrm{d})$ above. This then prevents the possibility that regular subpatches of cardinality $\leqq r$ join $T^{t_{1}} R^{\alpha} C$ to $B$ or $T^{t_{2}} R^{\beta} B$ to $C$, respectively. For instance, points $x_{3}$ and $x_{4}$ in Fig. 16, below, cannot belong to a regular subpatch of cardinality $\leqq r$, as is also the case for $x_{1}$ and $x_{2}$.)

Then the regular patch (see Fig. 16)

$$
\begin{aligned}
G_{2}= & G_{1} \cup C \cup T^{t_{2}} R^{\beta} G_{1} \cup T^{t_{1}} R^{\alpha}\left[C \cup T^{t_{2}} R^{\beta} A\right] \\
= & G_{1} \cup C \cup T^{t_{1}} R^{\alpha} C \cup T^{t_{2}} R^{\beta} B \cup T^{t_{2}} R^{\beta} A \cup T^{\left(t_{2}+R^{\beta}\left(t_{1}\right)\right)} R^{(\alpha+\beta)} A \\
& \cup T^{\left(t_{1}+R^{\alpha}\left(t_{2}\right)\right)} R^{(\alpha+\beta)} A
\end{aligned}
$$

contains twins and a cousin.

Step 2. So without loss of generality assume $\Omega$ contains twins of order $r$, namely $A, T^{t_{1}} A$, and a cousin $T^{t_{2}} R^{\beta} A$ with $\beta \in\left(-\frac{\pi}{100}, \frac{\pi}{100}\right)$. We will prove then that $V(S)$ admits triplets of order $r$. 


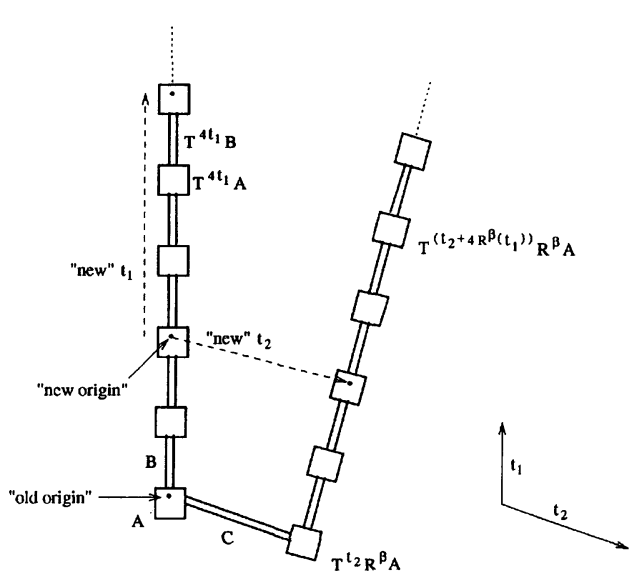

(a) $P_{n}$ for $\beta<0$

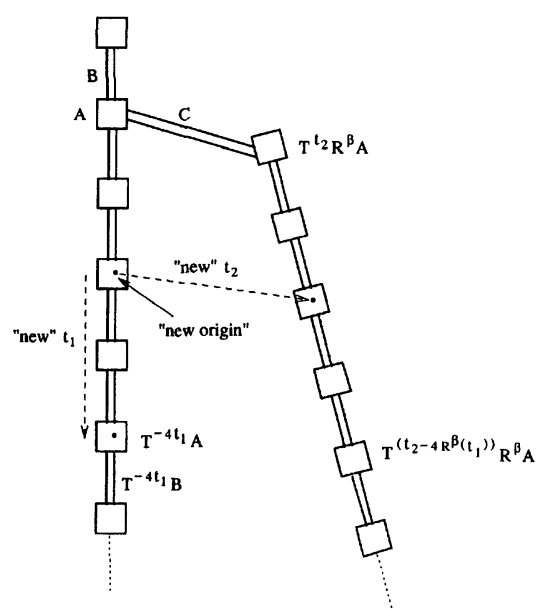

(b) $P_{n}$ for $\beta>0$

Fig. 17.

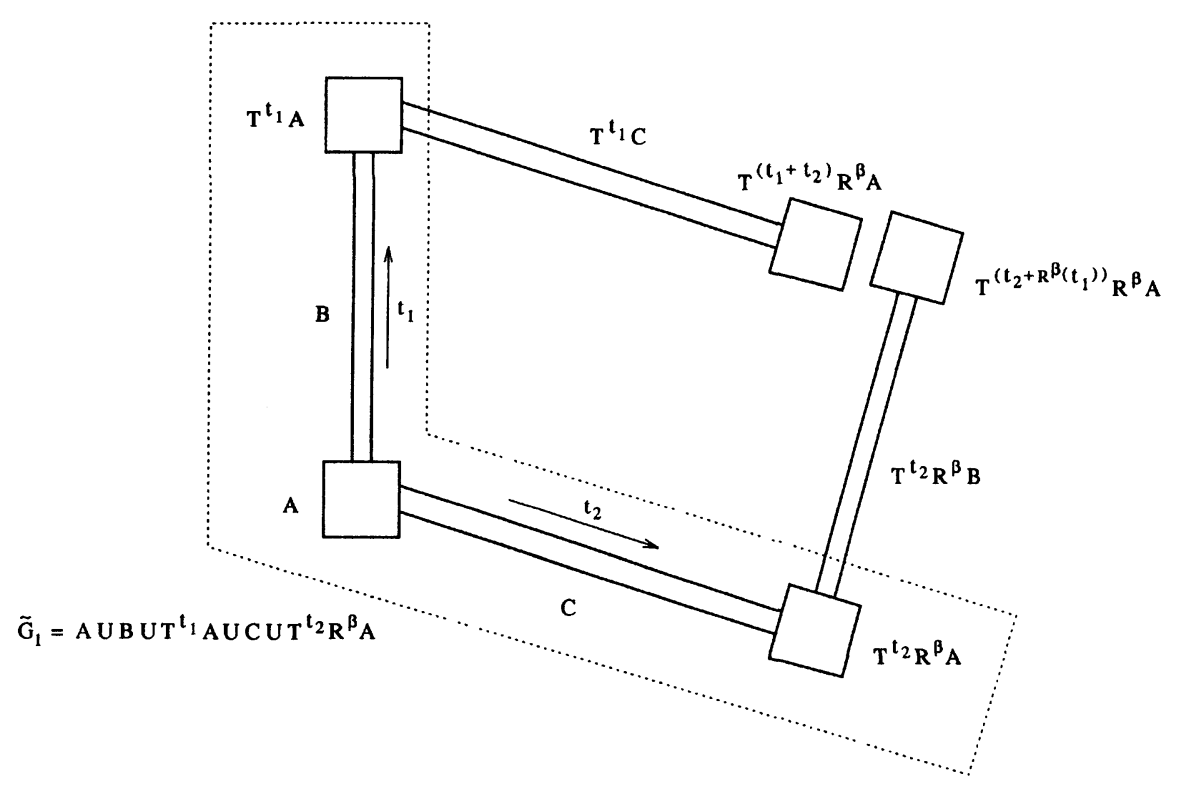

Fig. 18. $\tilde{G}_{2}$ 
If $\beta=0$ then $V(S)$ admits triplets of order $r$. But by the proof of Theorem 1 , Step 1, this contradicts the assumption that $S$ is an aperiodic protoset. Hence we can assume without loss of generality that $\beta \neq 0$.

Consider then sequence of regular patches (see Figs. 17 (a), (b))

$$
P_{n}=\bigcup_{i=0}^{n}\left\{T^{\operatorname{sgn}(\beta) i t_{1}}\left[A \cup B \cup T^{t_{1}} A\right]\right\} \bigcup_{j=0}^{n}\left\{T^{\left(t_{2}+\operatorname{sgn}(\beta) j R^{\beta}\left(t_{1}\right)\right)} R^{\beta}\left[B \cup T^{t_{1}} A\right]\right\} .
$$

From supposition (2) it follows that for all $n \in \mathbf{N}, P_{n} \subset \tilde{\Omega}_{n}$ for some $\tilde{\Omega}_{n} \in V(S)$. So we may assume without loss of generality that:

a) $\left\|t_{1}\right\|>[\operatorname{diam}(A)+n \Delta]$

b) $\left\|R^{\beta}\left(t_{1}\right)-t_{1}\right\|>2 \operatorname{diam}(A)$;

c) $\cos ^{-1}\left(t_{1} \cdot t_{2} /\left\|t_{1}\right\|\left\|t_{2}\right\|\right) \in\left(\frac{\pi}{2}, \frac{3 \pi}{4}\right)$.

Let $\tilde{G}_{1}=A \cup B \cup T^{t_{1}} A \cup C \cup T^{t_{2}} R^{\beta} A$, where $B$ and $C$ are chosen as in $G_{1}$ above. Then the regular patch (see Fig. 18)

$$
\begin{aligned}
\tilde{G}_{2}= & \tilde{G}_{1} \cup T^{t_{2}} R^{\beta}\left[B \cup T^{t_{1}} A\right] \cup T^{t_{1}}\left[C \cup T^{t_{2}} R^{\beta} A\right] \\
= & A \cup B \cup T^{t_{1}} A \cup C \cup T^{t_{2}} R^{\beta} B \cup T^{t_{1}} C \cup T^{t_{2}} R^{\beta} A \cup T^{\left(t_{2}+R^{\beta}\left(t_{1}\right)\right)} R^{\beta} A \\
& \cup T^{\left(t_{1}+t_{2}\right)} R^{\beta} A
\end{aligned}
$$

contains triplets and, by (2), belongs to some $\tilde{\Omega} \in V(S)$. Hence $V(S)$ admits triplets of order $r$. But by the proof of Theorem 1, step 1, this contradicts the assumption that $S$ is an aperiodic protoset. Hence, given $r>0$, deceptions of order $r$ exist.

Q.E.D.

\section{Concluding Remarks}

Aperiodic tiling models with (deterministic) local matching rules have been used successfully as models of the structure of quasicrystals. However we show that for a large class of such models, including all those that have been investigated thus far, the problem of guaranteeing the growth of perfect single grain quasicrystals of arbitrary large size is unavoidable; local matching rules alone (i.e., without added features such as probabilistic interactions) are insufficient to guarantee defect-free growth.

Acknowledgements. The authors are grateful to C. Radin for many interesting discussions and constant encouragement. We would also like to thank M. Senechal for her help and for bringing to the attention of the authors several useful references.

\section{References}

1. Shechtman, D., Blech, I.A., Gratias, D., Cahn, J.W.: Phys. Rev. Lett. 53, No. 20, 1951-1953 (1984)

2. Levine, D., Steinhardt, P.J.: Phys. Rev. Lett. 53, No. 34, 2477-2480 (1984)

3. Steinhardt, P.J., Ostlund, S.: The Physics of Quasicrystals. Singapore: World Scientific, 1987 
4. Jarić, M.V. (ed.): Aperiodicity and Order 1, Introduction to Quasicrystals. New York: Academic Press, 1988

5. Senechal, M.: Quasicrystals and Geometry. (to be published).

6. Elswifk, H.B., De Hosson, J.Th.M., van Smaalen, S., de Boer, J.L.: Phys. Rev. B 38, No. 3, 1681-1685 (1988)

7. Goldman, A.I. et al: Phys. Rev. Lett. 61, No. 17, 1962-1965 (1988)

8. Gardner, M.: Sci. Amer. 236, 110-121 (1977)

9. Onoda, G.Y., Steinhardt, P.J., DiVincenzo, D.P., Socolar, J.E.S.: Phys. Rev. Lett. 60, No. 25, 2653-2656 (1988)

10. Jarić, M.V., Ronchetti, M.: Phys. Rev. Lett. 62, No. 10, 1209 (1989)

11. Onoda, G.Y., Steinhardt, P.J., DiVincenzo, D.P., Socolar, J.E.S.: Phys. Rev. Lett. 62, No. 10, $1210(1989)$

12. Ingersent, K., Steinhardt, P.J.: Phys. Rev. Lett. 64, No. 17, 2034-2037 (1990)

13. Penrose, R.: In: Aperiodicity and Order 2, Introduction to the Mathematics of Quasicrystals. Jarić, M.V. (ed.). Boston: Academic Press, 1989, pp. 53-80

14. Socolar, J.E.S.: In: Quasicrystals: The State of the Art. DiVincenzo, D.P., Steinhardt, P.J. (eds.). Singapore: World Scientific, 1991

15. Grünbaum, B., Shephard, G.C.: Tilings and Patterns. New York: W.H. Freeman, 1987

16. Radin, C., Wolff, M.: Geom. Dedicata. 42, 355-360 (1992)

Communicated by J.L. Lebowitz 